\title{
Mobile Tourist Guide Services with Software Agents
}

\author{
Juan Pavón ${ }^{1}$, Juan M. Corchado ${ }^{2}$, Jorge J. Gómez-Sanz and Luis F. Castillo Ossa ${ }^{2}$ \\ ${ }^{1}$ Dep. Sistemas Informáticos y Programación \\ Universidad Complutense Madrid \\ Ciudad Universitaria $\mathrm{s} / \mathrm{n}$ \\ 28040, Madrid, Spain \\ ${ }^{2}$ Dep. Informática y Automática \\ Universidad de Salamanca \\ Plaza de la Merced s/n \\ 37008, Salamanca, Spain \\ Email: corchado@usal.es \\ http://gsii.usal.es/
}

\begin{abstract}
Applications for mobile devices have some restrictions because of the limited capabilities and heterogeneity of these devices. However, their communication capabilities allow the distribution of the application control and access to information in a network. If we also consider the changing environment when a user moves from one location to another, we should have software that is context-aware and able to adapt to new situations. Agent technology can support these requirements because of its distributed nature and the ability to combine flexible component architectures, some of them with planning and learning capabilities, which are appropriate for adapting to changing environments. In this paper we show one such application, a mobile tourist guide service, which has been built as a multi-agent system, where some agents are deliberative and combine the Beliefs-Desires-Intentions pproach with learning capabilities of Case Base Reasoning techniques.
\end{abstract}

Keywords. Mobile Services, Tourism Application Software, Software Agents, Ambience Intelligence, INGENIAS methodology

\section{Introduction}

Applications for mobile devices have some restrictions because of the limited capabilities (battery lifetime, processor and memory capacity, small display and keyboard) and heterogeneity of these devices. However, their size, portability, and communication capabilities provide new opportunities for new types of services. The 
telecommunication industry expects a new expansion with the development of UMTS and third generation mobile systems. New challenges of this field require a technology that facilitates the construction of more dynamic, intelligent, flexible and open applications, capable of working in a real time environment. Because of its distributed nature software agents are a promising approach for addressing these issues. Furthermore, when considering mobile services we should also be concerned about changes in the environment when a user moves from one location to another. Therefore, the software should be context-aware and able to adapt to new situations. Multi-agent systems can fit in changing environments, as they are built as a combination of flexible components, some of them with planning and learning capabilities.

In this paper we present a tourist guide service, which works on mobile devices, as an application of agent technology that addresses these issues. There are several types of agents in the system, some of them working in the mobile devices, essentially for interaction with the user, and others distributed in servers, which have advanced information processing and reasoning capabilities. One interesting fact concerning these second type of agents is the way they combine the Beliefs-Desires-Intentions (BDI) approach [7] with learning capabilities of Case Base Reasoning (CBR) techniques [2,3], which facilitates context-aware behaviour, that makes use of past experiences to find the best plans to achieve goals.

The next section describes the mobile tourist guide service and its main components, which are organized as a multi-agent system. The system is able to program a tourist route, and modify it according to the conditions of the places to visit and the available time for the tourist. Because of its design, the services of the tourist guide agent can be easily extended (e.g. to recommend restaurants in the area of the tourist route), and support a high degree of scalability in the number of users. Section 3 describes the agents in the system. Section 4 presents the results of the validation of the system by tourists of Salamanca who have used it. Finally, section 5 highlights some of the main contributions of this kind of systems for providing new services and considers some open issues.

\section{The Mobile Tourist Guide Service as a Multi-Agent System}

The Mobile Tourist Guide Service, called TOURIST GUIDE-USAL, provides a set of agents to assist potential tourists in the organization of their tourist routes and enable them to modify their schedules on the move using wireless communication systems. There is one assistant agent for each user of the system, the Performer Agent (GlezBedia et al., 2002). Each user (tourist) willing to use the system has to register and solicit one of these agents. A Performer Agent can then be installed in the mobile phone of the tourist and provide an interface such as the one depicted in Figure 1. To start using the system, the tourist has to provide a set of parameters, such as visiting period of time and a number of restrictions related to cost and tourist interest (e.g., monuments or architectonic style). 


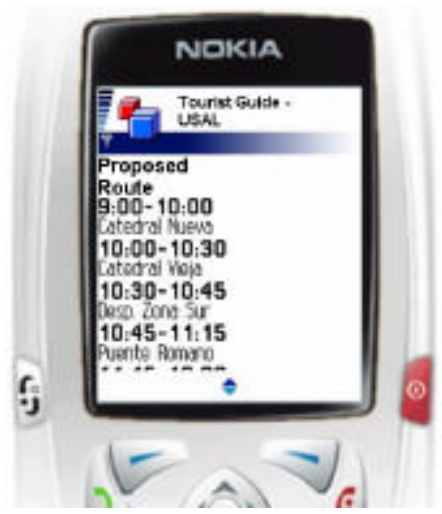

(a)

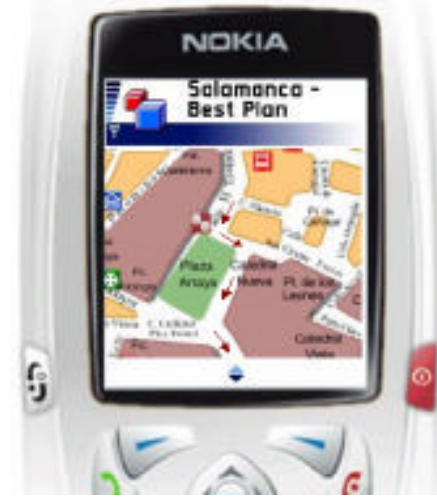

(b)

Figure 1. User interface to TOURIST GUIDE-USAL

Once the tourist has configured the preferences for a tour, the associated Performer Agent will contact with the Planner Agent, which assesses tourists and help them to identify tourist routes in the city taking into account their preferences. Planner Agents reside in network servers, as they have to process a considerable amount of information, both descriptive data and cases from past experiences. Planner Agents are supported by Tracker agents, which maintain updated information about the monuments, the restaurants, public transport conditions, etc. For instance, whether a monument is open for visitors, and whether there is a waiting queue. These agents maintain horizontally and vertically compiled information on hotel accommodation, restaurants, the commercial sector and transport, in order to meet the needs of the potential visitor on an individually customized basis, and respond to requests for information, reservations and purchases in the precise moment that they are expressed.

The design of the agent system has been done following the INGENIAS methodology [6] and using its supporting tools (the INGENIAS Development Kit, available at http://ingenias.sourceforge.net). Figure 2 shows that the agent organization, TOURIST GUIDE USAL, has two groups of agents. One is the group of agents which are deployed on the mobile devices and the other consists of those agents that run on servers. A Performer Agent can run either on the mobile device (if it has enough capabilities, and the user authorizes its installation), or in a server (in this case the interface with the user will be simpler, by using SMS, and limited to the display of routes and schedules in text form). The user may decide whether to install the corresponding Performer Agent on a mobile phone or PDA, or run it on the server and interact with it via its mobile device. The first choice supposes a reduction of the cost, since the tourist can interact with his agent as much as needed at no cost because it is installed in the wireless device. Nevertheless, in both cases the agent will have to contact regularly with the Planner Agent.

Tracker Agents will consult information about monuments in the corresponding information sources (e.g., web servers when available or by an update service, which 
should be maintained by the city tourist department). As we do not want to limit the type of information source, these are modelled as objects, for instance Monument_Information in Figure 2, which are wrapped by Tracker Agents, which are responsible of offering a normalized interface to Planner Agents. In this way we encapsulate the access to different types of information sources.

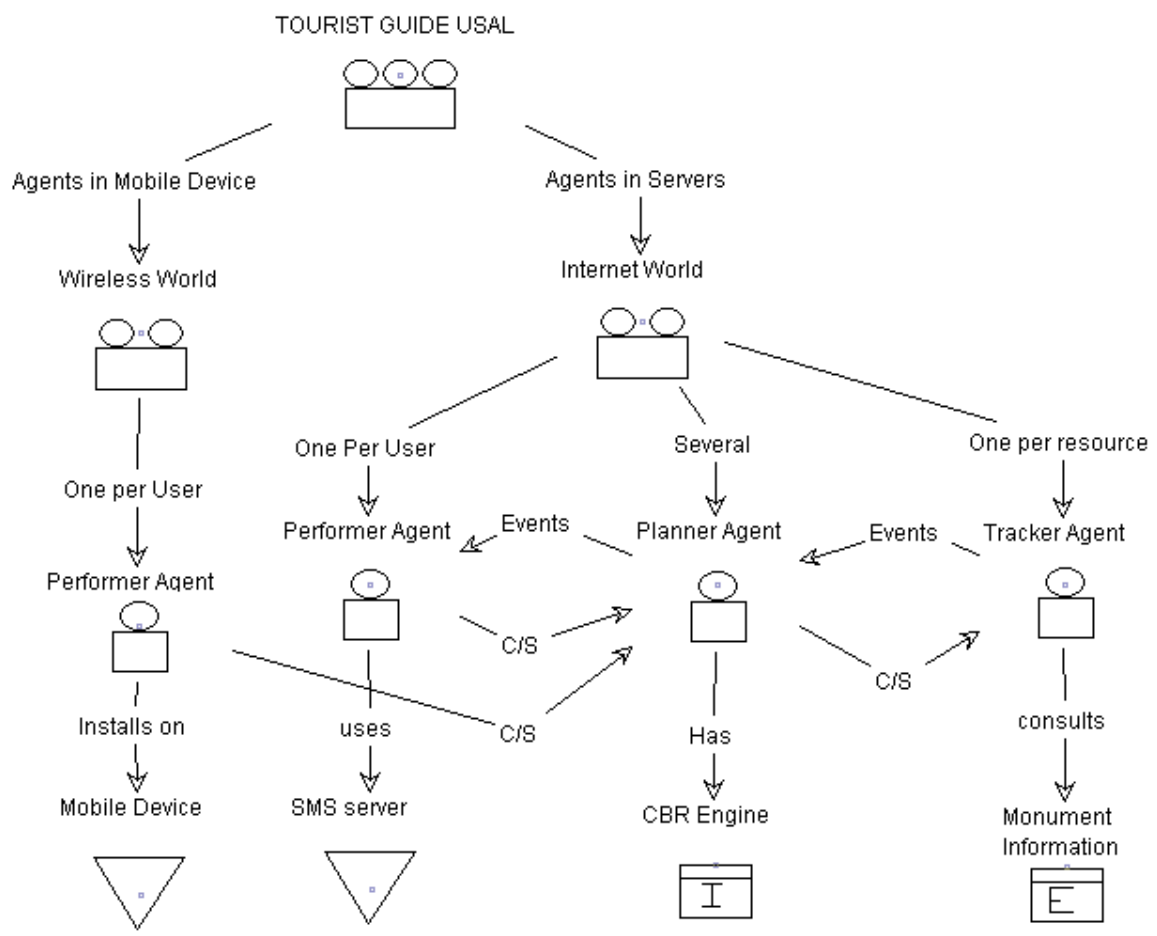

Figure 2. Organization of the TOURIST GUIDE USAL agent system

The Performer Agents interact with the Planner Agent looking for plans, and the Tracker Agent interacts with the Planner Agent to exchange information (a Planner Agent request for information to a Tracker Agent and can register for events; therefore, Client/Server and Event channel associations are established between these agents, as shown in Figure 2). The Planner agent is the only deliberative agent in this architecture, and has been built as a BDI agent that makes use of a CBR engine in order to be able to adapt to changing conditions by using past experience to decide which plans to propose. The Performer Agents can be considered assistant agents and the Tracker Agent is a reactive agent. 


\section{Agents Design}

Each agent is initially described by identifying its responsibilities (what goals an agent is compromised to pursue) and capabilities (what tasks is able to perform). The three agent types in the Mobile Tourist Guide System are represented in Figure 3 (Planner Agent), Figure 4 (Performer Agent), and Figure 5 (Tracker Agent).

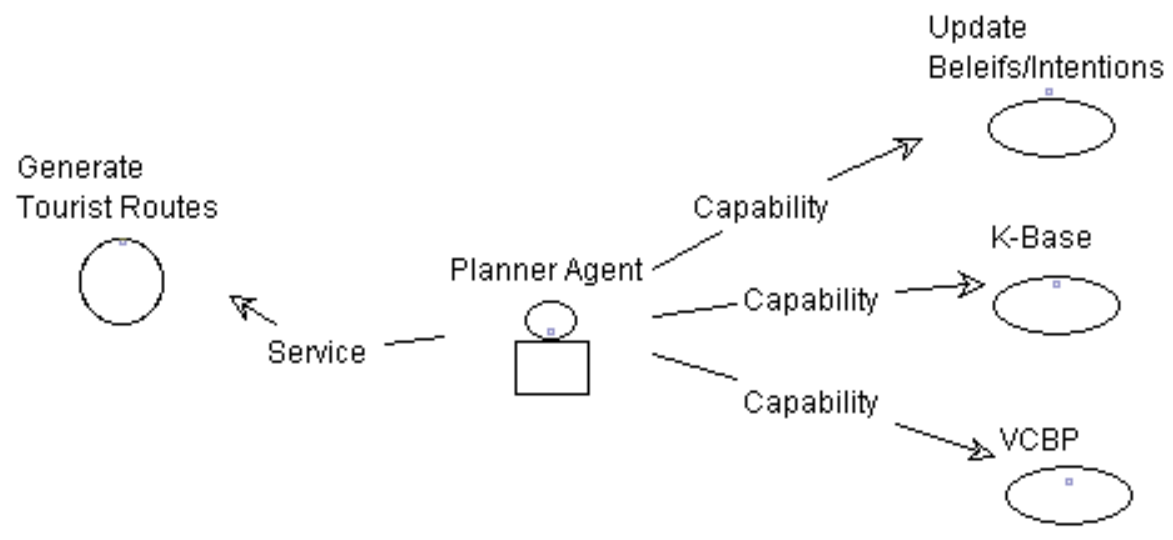

Figure 3. Planner Agent

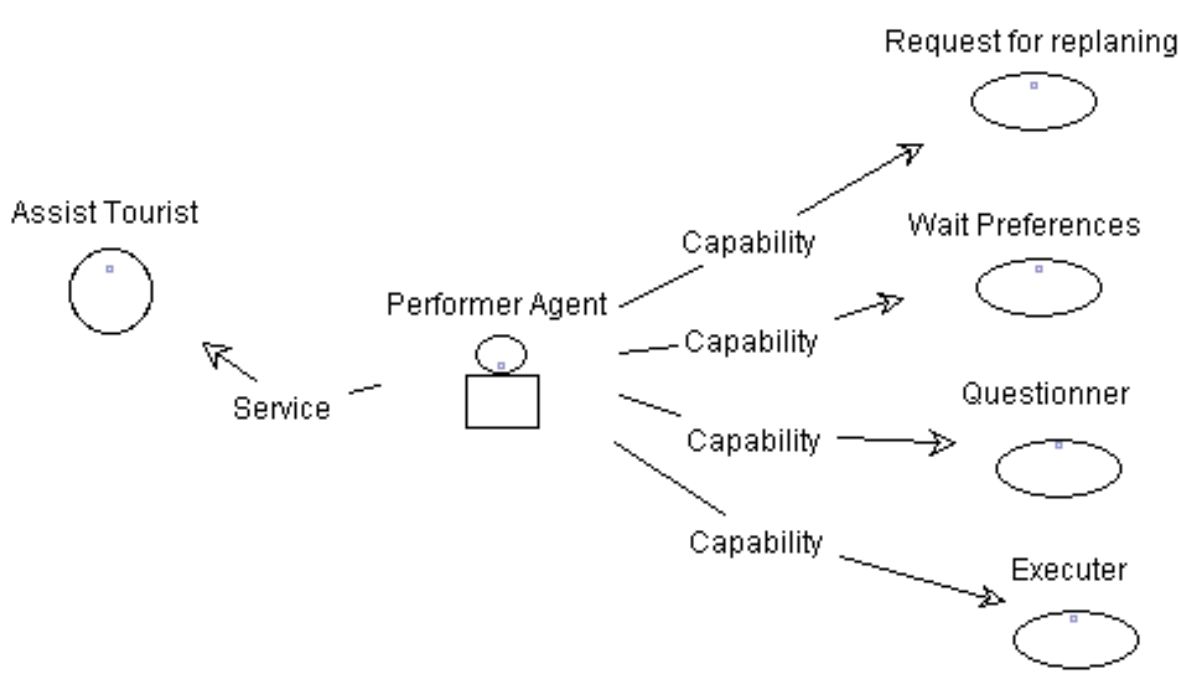

Figure 4. Performer Agent

The behavior of each agent is defined with three comp onents:

- Mental state, an aggregation of mental entities such as goals, beliefs, facts, and compromises. 
- Mental state manager, which provides for operations to create, destroy and modify mental entities.

- Mental state processor, which determines how the mental state evolves, and it can be described in terms of rules, planning, etc.

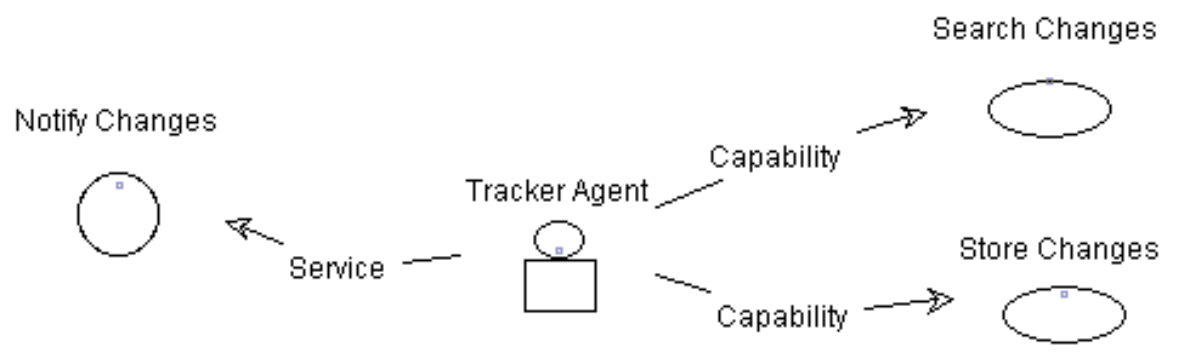

Figure 5. Tracker Agent

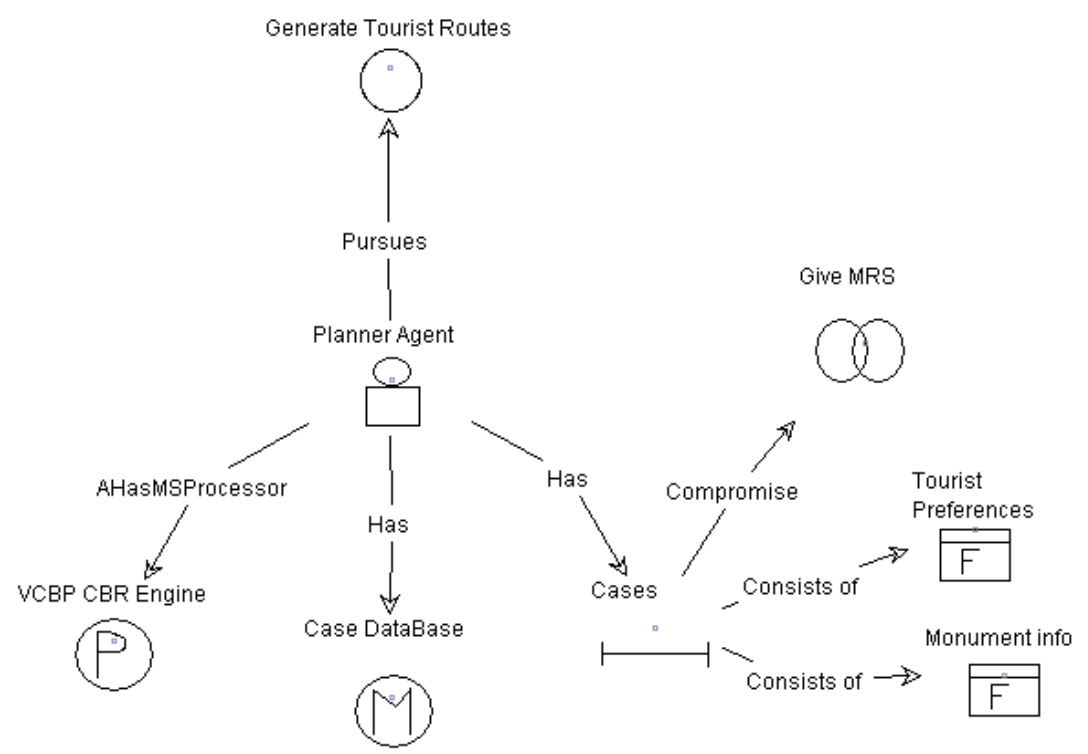

Figure 6. Planner Agent behaviour elements

Mental state can be seen as all the information that allows the agent to take decisions. This information is managed and processed in order to produce agent decisions and actions, by the mental state manager $(\mathrm{M})$ and processor $(\mathrm{P})$. The advantage of separating management and processing of mental state is the decoupling 
of the mechanis ms that implement the autonomy and intelligence of the agent from the conceptualization of the agent. In the case of the Planner Agent, Figure 6 shows the main mental state entities and the type of Mental state manager and processor. This agent is modeled using BDI concepts but the implementation relies on the use of a CBR engine. Therefore, mental state entities are regarded as cases. This is described in detail later in the paper.

The responsibility of the Planner Agent is to provide adequate plans to the Performer Agent given a number of conditions. This is represented as a goal Generate Tourist Route. This goal is refined in a goals/task diagram (Figure 7), which shows how the goal is broken down in sub-goals that can be achieved by execution of tasks. In this case, the goal involves the identification of those beliefs and intentions that can be used to generate a tourist route, and maintaining up-to-date those beliefs and intentions.

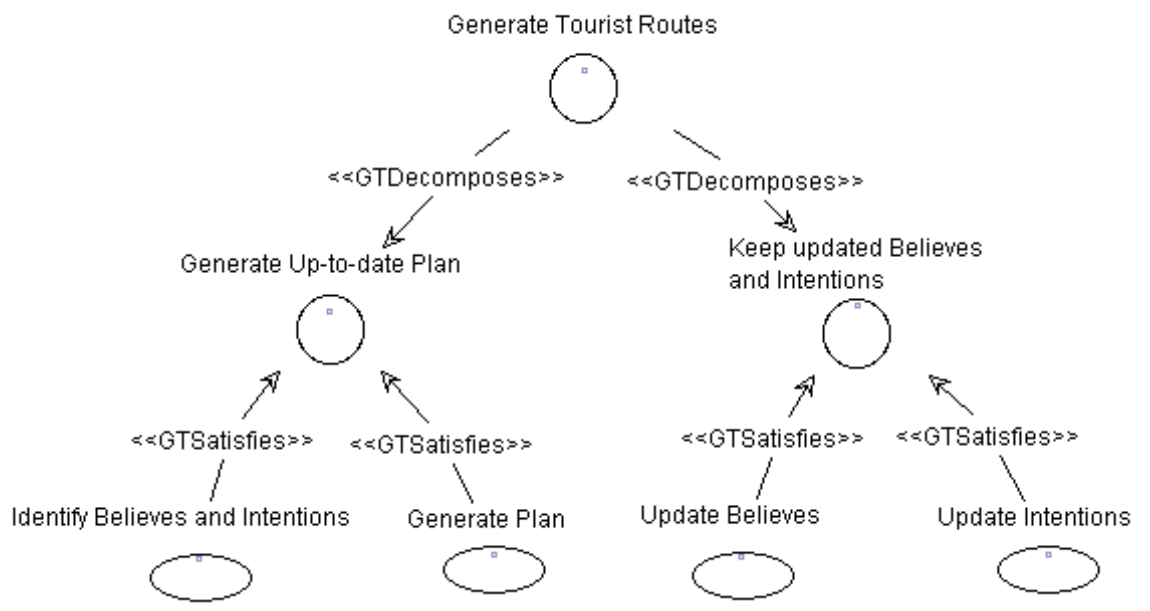

Figure 7. Goals/Tasks diagram for the Planner Agent

Some tasks require the collaboration of other agents through interactions, as the one defined in Figure 8, which shows how the Tracker Agent collaborates to keep track of changes in information about a monument. An interaction requires the identification of:

- Actors in the interaction: who plays the role of initiator and who are the collaborators. Which is the reason (goal) that motivates each actor to participate in the interaction.

- Definition of interaction units: messages, speech acts.

- Definition of the possible orders of interactions units: protocols.

- Which actions are performed in the interaction.

- Context of the interaction: which goal pursues the interaction and which are the mental states of its participants.

- Control model: coordination mechanisms. 
The interaction diagram in Figure 8 shows that the Tracker Agent initiates the interaction with a FIPA INFORM to notify of a change. The reason to do this is to satisfy its goal of providing the service NotifyChanges to registered agents. The Planner Agent participates in the interaction as collaborator, as it looks to keep updated its beliefs and intentions.

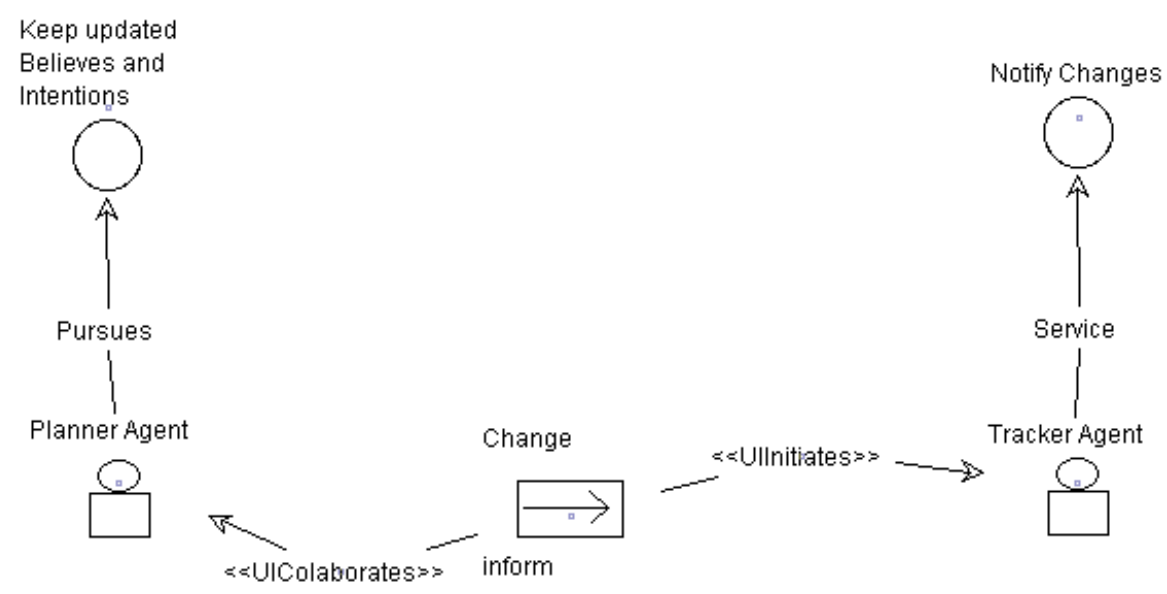

Figure 8. Interaction of Planner Agent with Tracker Agent

The rest of the goals and capabilities are worked out in a similar way. Each goal is broken down into subgoals, until they can be associated with concrete tasks. When they require cooperation with some other agent, the interaction is described, by considering the motivation. Interaction diagrams can be complemented with AUML sequence diagrams in order to show the temporal relationship between the interaction units.

An interesting design issue is the use of an hybrid BDI-CBR architecture for implementing Planner Agents, which are of deliberative nature and able to learn from past experience, thus adapting to changing context. The details of this are the subject of another paper [3].

\section{Evaluation results}

The proposed system has been used to improve an agent based system developed for guiding tourists around the city of Salamanca. As mentioned before, the tourists may use a mobile device to contact their agents and to indicate their preferences (monuments to visit, visits duration, time for dinner, amount of money to spend, etc.). There are different types of cases. The cases store information about the environment, for example the opening and closing times of monument. This type of information can be seen as an agent believe, for example, The Museum of Contemp orary Art opens form 9:00 to 14:00 and from to 16:30 to 20:00. Cases may also be previous successful 
routes (plans), which include the monuments to visit, the time to spend visiting each monument, information about the cost of the visit, the time required for going to one place to another, the characteristics of the route (museum route, family route, university route, roman route, gothic route, etc.), etc. Once a tourist contacts the system he has to describe his profile, to select the type visit in which he is interested in, to determine how much money he wants to spend and for how long, and the type of restaurants he, she or a family like more. This information is used to construct the problem case. Then the reasoning mechanism of the planning agent generates the plan, as described in [3].

The initial system, was tested from the $1^{\text {st }}$ of May to the $15^{\text {th }}$ of September 2003 . The case base was initially filled with information collected from the $1^{\text {st }}$ of February to the $25^{\text {th }}$ of June 2003. Local tourist guides provided the agent with a number of standard routes. Three hotels of the City offered the option to their 6217 guests to use the help of the agent or a professional tourist guide, $14 \%$ of them decided to use the agent based system and $23 \%$ of them used the help of a tourist guide. The rest of the tourists visited the city by themselves. During this period the Planner agent stored in its memory 1630 instances of tourist circuits, which covered a wide range of all the most common options that offers the City of Salamanca. The system was tested during 135 days and the results obtained were very encouraging. In this experiment the agent intentions were related to a one-day route (a maximum of 12 hours). On the arrival to the hotel the tourists were asked to evaluate their visit and the route. Table 1 shows the responses given by the tourists after their visit. The tourists that used the help of the agent-based tourist guide provided the answer directly to the agent.

\begin{tabular}{|l|c|c|c|c|c|c|}
\hline & $\%$ & \multicolumn{5}{|c|}{ Evaluation - degree of satisfaction } \\
\hline Tourists that... & & $8-10$ & $6-8$ & $4-6$ & $0-4$ & $\begin{array}{c}\text { No } \\
\text { answer }\end{array}$ \\
\hline Used the help of the agent & $14 \%$ & $(55,9 \%)$ & $(4,7 \%)$ & $(2,4 \%)$ & $(0,7 \%)$ & $(36,3 \%)$ \\
\hline $\begin{array}{l}\text { Used the help of a tourist } \\
\text { guide }\end{array}$ & $23 \%$ & $(62,7 \%)$ & $(19,6 \%)$ & $(8,9 \%)$ & $(1 \%)$ & $(7,8 \%)$ \\
\hline $\begin{array}{l}\text { Did not use any of the } \\
\text { previous }\end{array}$ & $63 \%$ & $(16,7 \%)$ & $(8,3 \%)$ & $(1,2 \%)$ & $(0,2 \%)$ & $(78,8 \%)$ \\
\hline
\end{tabular}

Table 1. Tourists evaluation

Table 1 shows the degree of satisfaction of the tourists. As it can be seen, the degree of satisfaction of the tourist that used the help of a professional tourist guide is higher that in the other two cases. Nevertheless, the percentage of the tourists whose degree of satisfaction was very high (between 8 and 10) is very similar in the case of the tourists that use the help of the agent and in the case of the tourists that use the tourist guide. $38 \%$ of the tourists that used the agent based system let us know that the system did not work successfully due to technical reasons (possibly the server was down, there was a lack of coverage, the tourist did not use the wireless system adequately, etc.) If we take this into consideration, we can say that most of the tourist $(92 \%)$ that used the help of the agent and did not have technical problems had a high 
or very high degree of satisfaction (6-10). This degree of satisfaction is higher that the one of the tourist $(82,3 \%)$ that used the help of a tourist guides.

\section{Conclusions}

The development of mobile services can benefit from the use of agents as they can easily distribute capabilities between user terminals and servers in a network. One important feature of mobile services is that they can be context-aware. As the user moves from one location to another, new information should be taken into account. This requires some adaptation mechanisms which are normally difficult to implement in a mobile phone because of its limited computing capabilities. In this sense, the availability of collaboration with agents in powerful servers is a solution. Furthermore, in an application as the one presented here, the user is new to the system, so there is no past experience from him. However, collective experience from other users in the system can be combined to get this knowledge.

The use of an agent development methodology (in this case, INGENIAS) has helped to build systematically the application. Agent related concepts, such as organization, goals, interactions, etc., can be useful to structure the distribution of responsibilities and capabilities.

Although distribution capabilities of agents are helpful, when dealing with mobile devices there is a need to consider failures in the connectivity. This requires caching of application data in the mobile device, which is complex task as the amount of data can be huge and vary dynamically.

\section{Acknowledgements}

This work has been supported by the Spanish Council for Research and Technology (MCYT) projects TEL99-0335-C04-03 and TIC2003-07369-C02-02.

\section{References}

1. Camacho D., Borrajo D. and Molina J. M. (2001) Intelligence Travell Planning: $a$ multiagent planing system to solve web problems in the e-turism domain. International Journal on Autonomous agens and Multiagent systems. 4(4) pp 385-390.

2. Corchado J. M. and Laza R. (2003). Constructing Deliberative Agents with Casebased Reasoning Technology, International Journal of Intelligent Systems. Vol 18, No. 12, December.

3. Corchado J. M. et al. (2004). Development of CBR-BDI Agents, submitted to the $7^{\text {th }}$ European Conference in Case-Based Reasoning, ECCBR 2004. 
4. Glez-Bedia M. and Corchado J. M. (2002) A planning strategy based on variational calculus for deliberative agents. Computing and Information Systems Journal. Vol 10, No 1, 2002. ISBN: 1352-9404, pp: 2-14.

5. Glez-Bedia M., Corchado J. M., Corchado E. S. and Fyfe C. (2002) Analytical Model for Constructing Deliberative Agents, Engineering Intelligent Systems, Vol 3: pp. 173185.

6. J. Pavón and J. J. Gómez-Sanz. Agent Oriented Software Engineering with INGENIAS. In: Multi-Agent Systems and Applications III, 3rd International Central and Eastern European Conference on Multi-Agent Sy stems, CEEMAS 2003. Lecture Notes in Computer Science 2691, Springer Verlag (2003) 394-403

7. Rao, A. S. and Georgeff, M. P. (1995). BDI Agents: From Theory to Practice. First International Conference on Multi-Agent Systems (ICMAS-95). San Franciso, USA. 